Artigo

\title{
Método de Ajuste Matemático para Validação de Dados de Sensores de Radiação de Onda Longa
}

\author{
Jose Dias Neto, José Celso Thomaz Júnior, Domingos Fernandes Urbano Neto \\ Laboratório de Instrumentação Meteorológica, Centro de Previsão de Tempo e Estudos \\ Climáticos, Instituto Nacional de Pesquisas Espaciais, Cachoeira Paulista, SP, Brasil.
}

Recebido: 18/2/2014 - Aceito: 20/5/2015

\begin{abstract}
Resumo
Uma metodologia para corrigir observações de Radiação de Onda Longa Descendente (OLD) geradas por estações de superfície é apresentada. Consiste na aplicação do método de mínimos quadrados para encontrar coeficientes $\left(\mathrm{k}_{\mathrm{s}}\right)$ específicos de sensores PIR Eppley (EP) e CGR4 Kipp \& Zonen (KZ) e reprocessamento das séries temporais. A avaliação da metodologia utilizou dados obtidos de um experimento realizado no Laboratório de Instrumentação Meteorológica - CPTEC/INPE. A aplicação de testes estatísticos na série de dados, com e sem correção, mostra uma redução significativa da Raiz do Erro Quadrático Médio, que passou de 9,02 a 0,43 W/m² para o sensor EP e de 4,11 a $1,21 \mathrm{~W} / \mathrm{m}^{2}$ para o KZ; o viés reduziu de $-7,99$ para $0,50 \mathrm{~W} / \mathrm{m}^{2}$ (EP) e de 3,53 para $0,27 \mathrm{~W} / \mathrm{m}^{2}$ (KZ). Um sensor CG4 (KZ) de referência calibrado no Physikalisch-Meteorologiches Observatorium Davos (PMOD), com rastreabilidade foi utilizado.
\end{abstract}

Palavras chave: radiação de onda longa, pirgeômetro.

\section{Mathematical Adjustment Method for Validation of Longwave Radiation Sensor Measurements}

\begin{abstract}
Hereby is presented a methodology to correct observations of Downwelling Longwave Radiation (DLR) generated by surface stations. It consists in the application of the least squares method to find coefficients $\left(\mathrm{k}_{\mathrm{s}}\right)$ of PIR Eppley (EP) and CGR4 Kipp \& Zonen (KZ) sensors, and reprocessing of time series. The evaluation of the methodology used data obtained in an experiment conducted in the Meteorological Instrumentation Laboratory - CPTEC / INPE. The application of statistical tests in the data series, with and without correction shows a significant reduction of the Root Mean Square Error, which went from 9,02 to $0,43 \mathrm{~W} / \mathrm{m}^{2}$ for the EP sensor and 4,11 to $1,21 \mathrm{~W} / \mathrm{m}^{2}$ for KZ; bias decreased from 0,50 to $-7,99 \mathrm{~W} / \mathrm{m}^{2}$ (EP) and 3,53 to $0,27 \mathrm{~W} / \mathrm{m}^{2}(\mathrm{KZ})$. A CG4 sensor (KZ) of the calibrated reference PhysikalischMeteorologiches Observatorium Davos (PMOD) with traceability was used.
\end{abstract}

Keywords: longwave radiation, pyrgeometer.

\section{Introdução}

As recentes mudanças no clima ressaltam a importância em monitorar o comportamento das variáveis ambientais (Trenberth et al., 2006), principalmente aquelas que controlam o balanço de energia do planeta, como as componentes da radiação atmosférica. As componentes da Radiação de Onda Longa Ascendente e Descendente (respectivamente OLA e OLD) desempenham um papel importante na determinação do saldo de radiação e do fluxo radiativo. Estudos observacionais indicam que estas com-

Autor de correspondência: Jose Dias Neto, jdiasn@gmail.com. ponentes são fundamentais para uma melhor compreensão dos fluxos de energia entre continente-atmosfera e oceanoatmosfera (Webster et al., 1992; Dickey et al., 1994). Harries et al. (2001) realizaram análise em série de dados da radiação de onda longa, observados no topo da atmosfera dentro da janela atmosférica, com duração de 30 anos, obtidas pelos satélites Nimbus 4 e ADEOS, mostraram que as mudanças no espectro de emissão foram causadas por alteração na concentração de espécies químicas responsáveis pelo efeito de estufa. Tett et al. (1999) apresentaram evidências de alterações da temperatura nas proximidades da superfície a partir de estudo realizado com dados obser- 
vados e simulações com o modelo acoplado HadCM2. Essas alterações foram explicadas como sendo resposta às alterações na concentração dos gases responsáveis pelo efeito de estufa.

O monitoramento in situ de radiação é fundamental para validação de estimativas por sensoriamento remoto ou de resultados de modelos numéricos ou teóricos/conceituais. No entanto, de acordo com o levantamento realizado pelo Instituto Nacional de Pesquisas Espaciais (INPE) e publicado no relatório "Solar and Wind Energy Resource Assessment" (Pereira et al., 2006) a base de dados de radiação de superfície necessária para a validação de modelos é extremamente deficiente no Brasil e em toda a América Latina. Diferente de outras propriedades como temperatura ou radiação de onda curta (ROC), as séries temporais de radiação de onda longa descendente (OLD) apresentam qualidade discutível por não haver procedimentos de avaliação e controle de qualidade bem estabelecidos.

No Brasil, o Sistema de Organização Nacional de Dados Ambientais (SONDA), que visa aprimorar a base de dados de superfície necessária ao levantamento dos recursos de energia solar e eólica no Brasil e consequente planejamento de seu uso (Thomaz, 2012), mantém uma rede de estações solarimétricas/anemométricas distribuídas pelo país, gerando séries temporais desde 2003. Três sítios observacionais foram equipados com sensores para medida de OLD (pirgeômetros), fabricados pela Eppley Laboratory, Inc. - EPLAB. Em fase de expansão, o SONDA passou a utilizar nos últimos anos pirgeômetros fornecidos pela Kipp \& Zonen. Contudo, para garantir a confiabilidade dos dados gerados é necessário avaliar as séries temporais de OLD fornecidas pelos diferentes sensores, assim como avaliar o impacto causado nos dados pela mudança de modelos de pirgeômetros ao longo do tempo.

Entretanto, não existe uma metodologia definida e consolidada na comunidade científica que esteja disponível para realizar essas análises e correções, principalmente quando pretende-se corrigir dados pretéritos depositados em bancos de dados. Desta forma, o propósito deste trabalho é apresentar uma metodologia capaz de realizar estimativas dos coeficientes dos pirgeômetros para que correções em séries temporais de Radiação de Onda Longa Descendente sejam realizadas.

\section{Materiais e Métodos}

Esta seção está dividida em três partes na qual serão apresentados 1) os aspectos teóricos para obtenção das equações de quantificação da OLD para os sensores da Eppley e da Kipp \& Zonen; 2) o experimento utilizado para aquisição dos dados experimentais e 3) o sistema de equações empregado para obtenção dos coeficientes e as equações empregadas durante a avaliação dos dados.

\subsection{Aspectos teóricos}

A Figura 1 ilustra a estrutura de um sensor de OLD e as componentes do fluxo envolvidas no balanço. O instrumento é composto por uma caixa que pode ser metálica (bronze) ou plástica para abrigar os componentes eletrônicos. Dentro da caixa localiza-se uma termopilha coberta por um domo de sílica que possui função dupla de proteger e filtrar interferências de ondas com o comprimento de onda fora do intervalo de 3,5 à $50 \mu \mathrm{m}$ para o sensor PIR da Eppley e de 4,5 à $42 \mu \mathrm{m}$ para o sensor CG4/CGR4 da Kipp $\&$ Zonen. São pequenas as diferenças técnicas-observacionais entre os sensores da Eppley e Kipp \& Zonen (daqui em diante referidos com EP e KZ, respectivamente). A diferença mais importante é a ausência de um termistor no domo do sensor KZ enquanto que no pirgeômetro EP existem termistores para medir a temperatura no domo e no corpo do sensor (Fig. 1).

O balanço do fluxo radiativo $F_{\text {net }}$ na termopilha dos pirgeômetros, seguindo o formalismo apresentado por

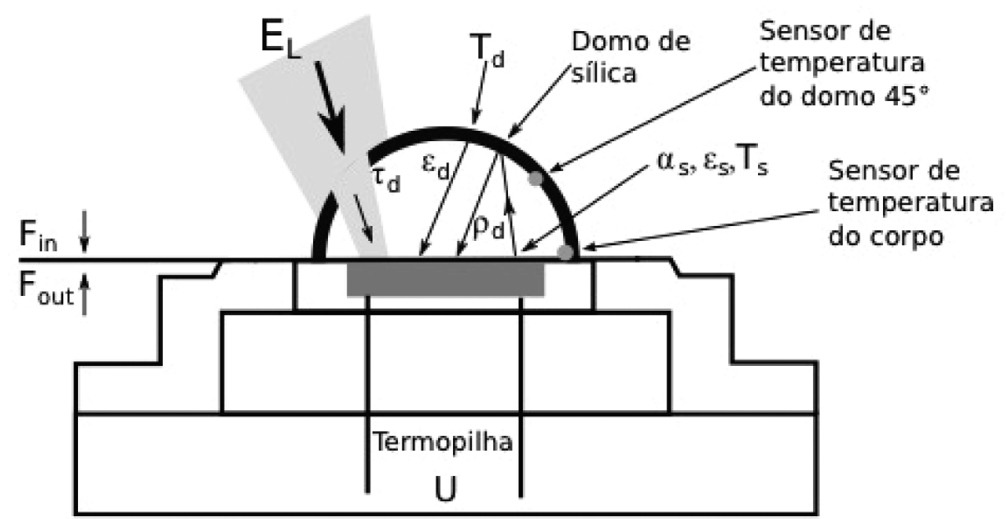

Figura 1 - Representação esquemática da estrutura de um pirgeômetro. (adaptado de Philipona et al., 1995). $\alpha_{d}$ é a absortância do domo, $\tau_{d}$ é a transmitância do domo, $\rho_{d}$ é a reflectância do domo, $T_{d}$ é a temperatura do domo, $\alpha_{s}$ é a absortância da termopilha, $\varepsilon_{s}$ é a emissividade da termopilha, $T_{s}$ é a temperatura da superfície da termopilha, $E_{L}$ é irradiância da onda longa (L é igual a EP para o sensor da Eppley e igual a KZ), $\mathrm{F}_{\text {in }}$ é o fluxo que incide sobre a termopilha e $\mathrm{F}_{\text {out }}$ é o fluxo emitido pela termopilha . 
Philipona et al. (1995), é descrito pela diferença entre o fluxo incidente $F_{\text {in }}$ e o fluxo emitido $F_{\text {out }}$ :

$$
F_{\text {net }}=F_{\text {in }}-F_{\text {out }}
$$

O fluxo $F_{\text {out }}$, para pirgeômetros EP e KZ, é determinado pela equação:

$$
F_{\text {out }}=\varepsilon_{s} \sigma T_{s}^{4}
$$

onde $\varepsilon_{s}$ é a emissividade da termopilha, $\sigma$ é a constante de Stefan-Boltzmann, e $T_{s}$ é a temperatura da superfície da termopilha.

As equações que descrevem o fluxos incidentes na termopilha são particulares para cada um dos instrumentos ( $F_{\text {inEP }}$ para o sensor EP $F_{\text {inKZ }}$ para o sensor KZ), e são calculados respectivamente por:

$$
\begin{aligned}
& F_{\text {inEP }}=\alpha_{s} \tau_{d} E_{L}+\alpha_{s} \varepsilon_{d} \sigma T_{d}^{4}+\alpha_{s} \rho_{d} \varepsilon_{s} \sigma T_{s}^{4} \\
& F_{i n K Z}=\alpha_{s} \tau_{d} E_{L}+\alpha_{s} \rho_{d} \varepsilon_{s} \sigma T_{s}^{4}
\end{aligned}
$$

onde $\tau_{d}$ é a transmitância do domo, $\rho_{d}$ é a reflectância do domo, $\alpha_{s}$ é a absortância da termopilha, $\varepsilon_{s}$ é a emissividade da termopilha (pela a lei de Kirchhoff após a termopilha atingir o equilíbrio termodinâmico $\alpha_{s}$ é igual a $\varepsilon_{s}$ ), $\sigma$ é a constante de Stefan-Boltzmann, $E_{L}$ (L é EP ou KZ) é irradiância da Onda Longa Descendente, $T_{d}$ é a temperatura do domo, fornecida pelo termistor do domo. A Equação (4) difere da Eq. (3) devido à ausência do termistor do domo no pirgeômetro KZ.

As irradiâncias medidas pelos sensores EP e KZ podem ser deduzidas da medida da diferença de potencial $U$ produzida pela termopilha e das equações (1) a (4) (Philipona et al., 1995) obtendo-se respectivamente as equações (5) e (6) (Dias Neto, J., 2014).

$$
\begin{aligned}
& E_{E P}=\frac{U}{C}\left(1+k_{1} \sigma T_{b}^{3}\right)+k_{2} \sigma T_{b}^{4}-k_{3} \sigma\left(T_{d}^{4}-T_{b}^{4}\right) \\
& E_{K Z}=\frac{U}{C}\left(1+k_{1} \sigma T_{b}^{3}\right)+k_{2} \sigma T_{b}^{4}
\end{aligned}
$$

onde $U$ é a diferença de potencial produzida pela termopilha, $C$ é a constante de sensibilidade do pirgeômetro, $T_{b}$ é a temperatura do corpo do sensor, $k_{1}, k_{2}$ e $k_{3}$ são os coeficientes de ajuste e necessitam ser determinados em laboratório. A equação (5) foi originalmente obtida por Philipona et al. (1995) a partir das equações (1), (2) e (3), onde os coeficientes obtidos são: $k_{1}=4 C \gamma \frac{1-\rho_{d} \alpha_{s}}{c \tau_{d}}, k_{2}=\frac{1-\rho_{d} \alpha_{s}-\varepsilon_{d}}{\tau_{d}} \mathrm{e}$ $k_{3}=\frac{\varepsilon_{d}}{\tau_{d}}$. A equação (6) foi obtida seguindo os mesmos passos adotados por Philipona et al. (1995) empregando as equações (1), (2) e (4). Os coeficientes encontrados são: $k_{1}=4 C \gamma \frac{1-\rho_{d} \alpha_{s}}{c \tau_{d}}$ e $k_{2}=\frac{1-\rho_{d} \alpha_{s}}{\tau_{d}}$. Estes coeficientes $k_{s}$ estão relacionados com as propriedades dos materiais empregados na confecção dos sensores e independem da direção da OLD (ascendente e descendente).

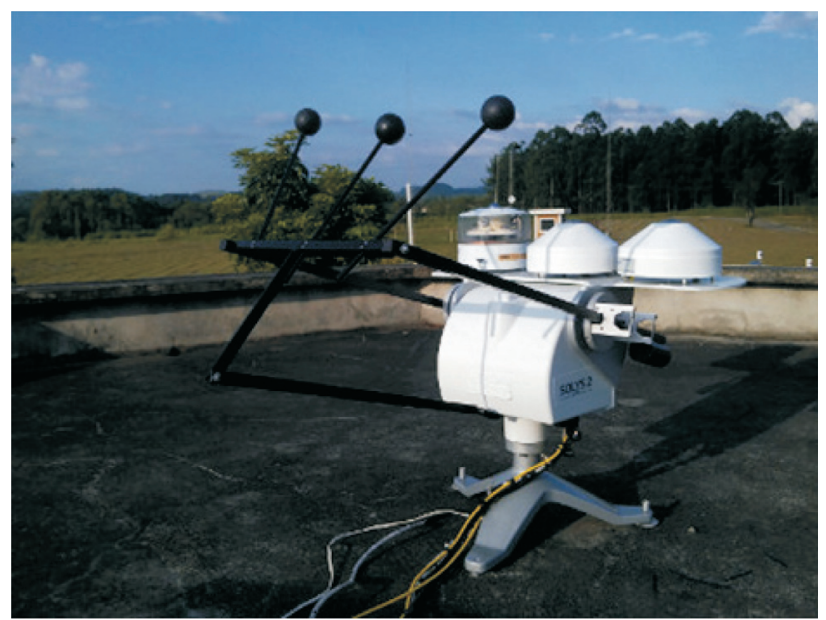

Figura 2 - Foto do aparato experimental montado no Laboratório de Instrumentação Meteorológica (LIM/CPTEC) composto pelos sensores: PIR da Eppley (esquerda), CG4 usado como padrão/referência (centro) e CGR4 (direita). Os sensores estão instalados no rastreador Solar Solys II, em que possui três braços com esferas na extremidade para sombrear os sensores conforme o posicionamento do sol.

\subsection{Campanha experimental}

Séries temporais de amostragens comparativas foram obtidas a partir da montagem de um aparato experimental nas instalações do Laboratório de Instrumentação Meteorológica (LIM) do Centro de Previsão de Tempo e Estudos Climáticos (CPTEC/INPE), na posição com latitude $22^{\circ} 41^{\prime} \mathrm{S}$, longitude $45^{\circ} 00^{\prime} \mathrm{W}$ e altitude $574 \mathrm{~m}$ (Fig. 2).

O pirgeômetro CG4 da Kipp \& Zonen, por ter sido calibrado no Phykalisch-Meteorologiches Observatorium Davos (PMOD), foi definido como o sensor de referência (REF). Dois pirgeômetros foram adicionados a esta estrutura para comparação: um sensor PIR (EP) que corresponde aos sensores que são atualmente empregados na rede SONDA e um sensor CGR4 (KZ) que corresponde aos novos sensores adquiridos pela rede (Tabela 1). A saturação do sinal resultante nesses sensores é um problema que pode ser causado pelo aquecimento devido à incidência da radiação solar direta pelo mau sombreamento domo (Philipona et al., 1995; Albrecht e Cox, 1977). Para evitar esse problema, os três sensores foram montados em um rastreador solar Solys II da Kipp \& Zonen e o sombreamento foi aplicado aos mesmos (Fig. 2). Sistemas de venti-

Tabela 1 - Sensores utilizados no experimento observacional. KZ é a sigla para o fabricante Keep \& Zonnen e EP para Eppley Laboratory, Inc.

\begin{tabular}{lccc}
\hline Sensor & Fabricante & Modelo & Número de série \\
\hline 1 & KZ & CG4 & $050478^{*}$ \\
2 & KZ & CGR4 & 120512 \\
3 & EP & PIR & $33495 \mathrm{~F} 3$ \\
\hline
\end{tabular}

*Sensor calibrado com rastreabilidade PMOD. 
lação foram utilizados para evitar o aquecimento não uniforme do corpo do instrumento (Philipona et al., 1995). Uma unidade de processamento e armazenamento de dados (datalogger) modelo CR3000 fornecida pela Campbell Scientific, Inc foi configurada para coletar dados na frequência de $1 \mathrm{~Hz}$ e armazenar as médias a cada minuto.

As séries temporais utilizadas neste trabalho foram coletadas entre os dias 130 e 220 de 2013 (10 de Maio a 10 de Agosto de 2013) totalizando 91 dias. Para que os coeficientes $k_{1}, k_{2}$ e $k_{3}$ fossem calculados os dados tiveram que obedecer os critérios estabelecidos pela Baseline Surface Radiation Network (McArthur, 2005). Os intervalos de dados que obedeceram a estes critérios foram classificados na categoria 'estável' pois também apresentaram o comportamento de uma curva suave (comportamento associado ao ciclo diurno) após calcular-se os valores médios durante 60 minutos de medidas. A Figura 3 ilustra os dois comportamentos, estável e instável, do dia 185 até 190, que corresponde a um intervalo estável e do dia 190 ao dia 205 corresponde a um intervalo instável.

O período estável compreendido entre os dias 185 e 190 foi utilizado para o cálculo dos novos coeficientes $k_{s} \mathrm{e}$ foi definido como Período Estável para Cálculo (PEC). Na Figura 4 encontra-se as curvas de dados para os sensores REF, EP e KZ.

Com o intuito de verificar se os coeficientes encontrados no PEC aplicam-se para uma data anterior bem como para uma data posterior, dois outros períodos estáveis foram selecionados. O Período Estável para Avaliação 1 (PEA 1) (Figura 5a) e o Período Estável para Avaliação 2

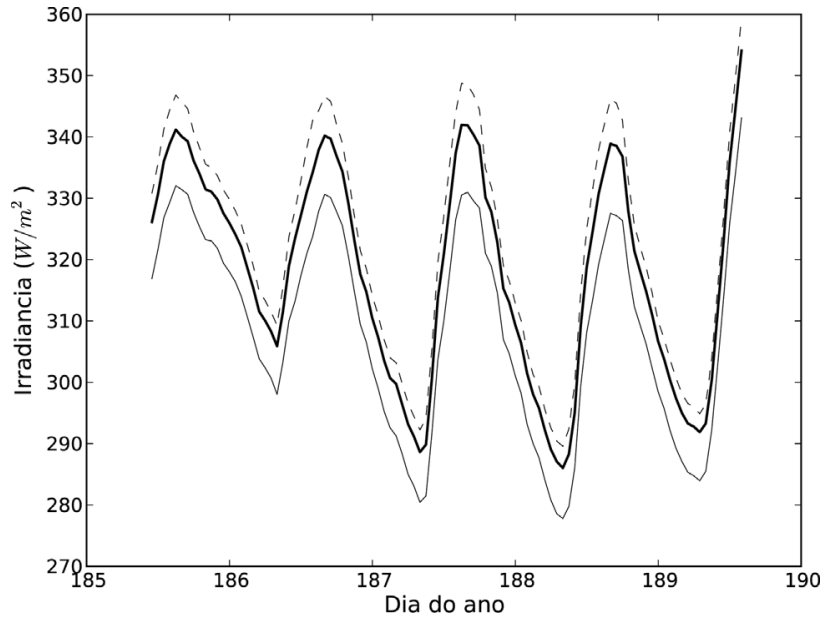

Figura 4 - Período estável de irradiância em W/m² (PEC) selecionado para calcular os coeficientes. Os dados obtidos pelo sensor REF (linha espessa), pelo sensor KZ (tracejado) e pelo sensor EP (linha delgada).

Figura 3 - Período instável de Irradiância selecionado para avaliação (PIA), em W/m². Dados obtidos pelo sensor REF (linha espessa), pelo sensor KZ (tracejado) e pelo sensor EP (linha delgada).

a) PEA 1

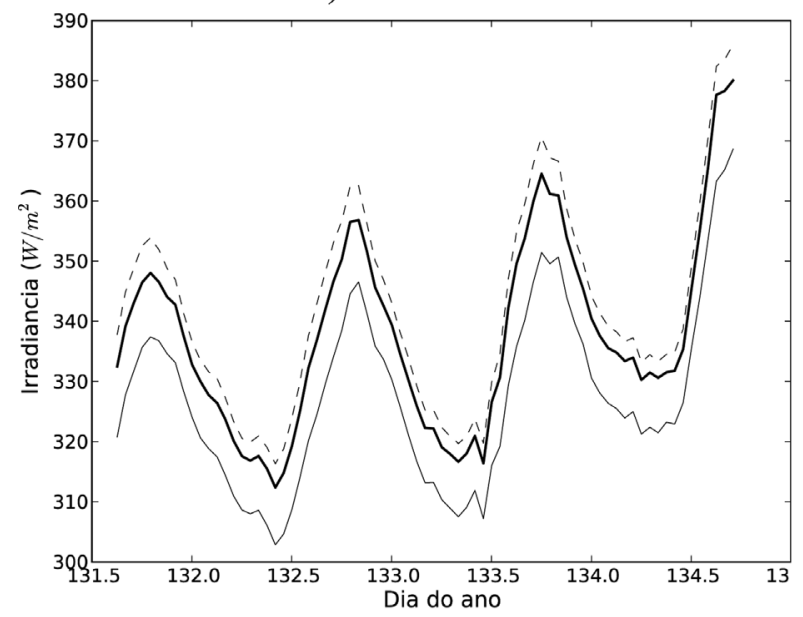

b) PEA 2

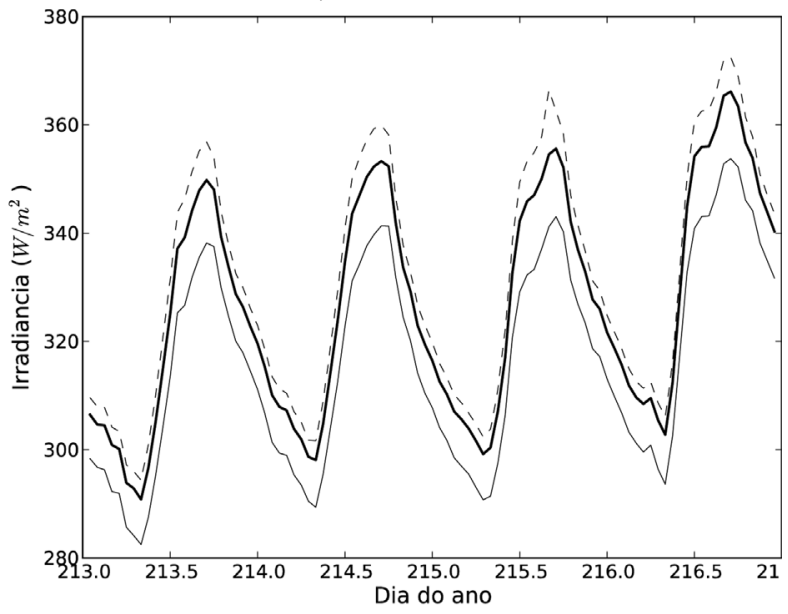

Figura 5 - Períodos estáveis de irradiância em W/m² obtidos pelo sensor REF linha espessa), pelo sensor KZ (linha tracejada) e pelo sensor EP (linha delgada). a) Período estável para avaliação pretérito ao PEC (PEA 1), e b) Período estável para avaliação posterior ao PEC (PEA 2). 
Tabela 2 - Períodos selecionados, duração.

\begin{tabular}{lccc}
\hline Períodos & Intervalos & Duração (dias) & Siglas \\
\hline Estável & 131 a 135 & 4 & PEA 1 \\
& 185 a 189 & 4 & PEC \\
& 213 a 217 & 4 & PEA 2 \\
Instável & 185 a 204 & 20 & PIA \\
\hline
\end{tabular}

(PEA 2) (Figura 5b), respectivamente anterior e posterior ao PEC (Tabela 2).

Para verificar se os coeficientes aplicam-se para uma série qualquer, foi selecionado um período instável de 20 dias, definido como Período Instável para Avaliação (PIA). Note que este período inclui o período estável PEC. A avaliação realizada com o período PIA é necessária, pois simula uma série de dados de OLD típica, ou seja, na maior parte do tempo tem um comportamento instável com alguns curtos períodos estáveis.

\subsection{Determinação dos coeficientes de ajuste}

Para determinar os coeficientes, aplica-se o Método de Mínimos Quadrados (MMQ) (Kreyszig, 2006) às equações (5) e (6), obtendo um sistema de equações para cada instrumento:

Sistema de equações para EP:

$$
\left\{\begin{array}{l}
\sum_{j=1}^{n} \frac{U_{j}}{C} T_{b j}^{3}\left(E_{p j}-\frac{U_{j}}{C}\right)=k_{1} \sum_{j=1}^{n}\left(\frac{U_{j}}{C}\right)^{2} \sigma T_{b j}^{6}+k_{2} \sum_{j=1}^{n} \frac{U_{j}}{C} \sigma T_{b j}^{7}+k_{3} \sum_{j=1}^{n} \sigma\left(T_{d j}^{4}-T_{b j}^{4}\right) T_{b j}^{3} \frac{U_{j}}{C} \\
\sum_{j=1}^{n} T_{b j}^{4}\left(E_{p j}-\frac{U_{j}}{C}\right)=k_{1} \sum_{j=1}^{n} \sigma T_{b j}^{7}+k_{2} \sum_{j=1}^{n} \sigma T_{b j}^{8}+k_{3} \sum_{j=1}^{n} \sigma\left(T_{d j}^{4}-T_{b j}^{4}\right) T_{b j}^{4} \\
\sum_{j=1}^{n}\left(E_{p j}-\frac{U_{j}}{C}\right)\left(T_{d j}^{4}-T_{b j}^{4}\right)=k_{1} \sum_{j=1}^{n} \frac{U_{j}}{C} \sigma T_{b j}^{3}\left(T_{d j}^{4}-T_{b j}^{4}\right)+k_{2} \sum_{j=1}^{n} \sigma T_{b j}^{4}\left(T_{d j}^{4}-T_{b j}^{4}\right)+k_{3} \sum_{j=1}^{n} \sigma\left(T_{d j}^{4}-T_{b j}^{4}\right)^{2}
\end{array}\right.
$$

Sistema de equações para KZ:

$$
\left\{\begin{array}{l}
\sum_{j=1}^{n}\left(E_{p j}-\frac{U_{j}}{C}\right) T_{b j}^{3}=k_{1} \sum_{j=1}^{n} \frac{U_{j}}{C} \sigma T_{b j}^{6}+k_{2} \sum_{j=1}^{n} \sigma T_{b j}^{7} \\
\sum_{j=1}^{n}\left(E_{p j}-\frac{U_{j}}{C}\right) T_{b j}^{4}=k_{1} \sum_{j=1}^{n} \frac{U_{j}}{C} \sigma T_{b j}^{7}+k_{2} \sum_{j=1}^{n} \sigma T_{b j}^{8}
\end{array}\right.
$$

Nos sistemas (7) e (8), $n$ é o número de amostragens colecionadas durante o período PEC. $E_{p}$ é a irradiância fornecida pelo sensor padrão. $T_{b}, T_{d}, U$ e $C$ são valores fornecidos pelos sensores em verificação. Os sistemas são resolvidos numericamente de modo a encontrar os valores $k_{1}, k_{2}$ e $k_{3}$, que são os coeficientes $k_{s}$.

A qualidade das estimativas de KZ e EP foi avaliada pela análise de parâmetros estatísticos como viés (VIES), raiz quadrada do erro quadrático médio (REQM), erro médio absoluto (EMA) e erro percentual relativo médio (EPMA) (Duarte et al., 2006). Os cálculos são feitos aos pares confrontando as medidas oriundas do sensor padrão $Y_{t}$ com aquelas fornecidas pelos sensores em verificação $y_{t}$, no caso KZ e EP, de acordo com as equações (9) a (12):

$$
\begin{gathered}
\text { VIES }=\frac{\sum_{t=1}^{n}\left(y_{t}-Y_{t}\right)}{n} \\
\text { REQM }=\sqrt{\frac{\sum_{t=1}^{n}\left(y_{t}-Y_{t}\right)^{2}}{n}}
\end{gathered}
$$

$$
\begin{gathered}
E M A=\frac{\sum_{t=1}^{n}\left|y_{t}-Y_{t}\right|}{n} \\
E P M A=\frac{100}{n} \sum_{t=1}^{n}\left|\frac{y_{t}-Y_{t}}{Y_{t}}\right|
\end{gathered}
$$

\section{Resultados e Discussão}

Foram utilizados dois pirgeômetros para avaliação, sendo um EP e um KZ. O sensor EP utiliza coeficientes estipulados pela Rede SONDA enquanto o KZ utiliza valores fornecidos pelo fabricante (Kipp \& Zonen). Estes coeficientes estão apresentados na Tabela 3 , juntamente com os valores de $k_{s}$ calculados através da metodologia descrita na Seção 2. Os valores $k_{1}$ e $k_{2}$ apresentam diferenças na segunda casa decimal. Para $k_{3}$, os valores diferem na casa da unidade. Coeficientes $k_{3}$ estão ausentes na Tabela 3 pois os pirgeômetros $\mathrm{KZ}$ não apresentam o termistor instalado no domo do instrumento.

Tabela 3 - Coeficientes de ajuste empregados na rede SONDA e do fabricante (Fab) versus coeficientes calculados (Calc) pelo método proposto

\begin{tabular}{lcccc}
\hline & $k_{s}$ & $k_{1}$ & $k_{2}$ & $k_{3}$ \\
\hline PIR 33495F3 & SONDA & 0,0530 & 1,0000 & 3,6500 \\
& Calc & 0,0223 & 1,0163 & 2,4833 \\
CGR4 120512 & Fab & 0 & 1 & - \\
& Calc & 0,0387 & 1,0008 & - \\
\hline
\end{tabular}


Os coeficientes $k_{s}$ são determinados durante o período estável PEC, sendo a primeira avaliação estatística entre os sensores em avaliação e o padrão, aplicada para este período. Esta análise avalia se o período escolhido (PEC) foi adequado para que os coeficientes sejam eficientes. A Tabela 4 mostra que o viés entre o sensor EP utilizando os coeficientes $k_{s}$ empregados pelo projeto SONDA e o sensor padrão passou de $-8,95$ para -0,002 após a utilização dos coeficientes calculados. Para o caso do sensor KZ, a redução do viés foi de 4,53 para -0,003. A REQM passou de $9,02(4,71)$ para $0,43(0,31)$ para o sensor EP (KZ). Estes valores mostram que o PEC escolhido foi adequado e gerou coeficientes capazes de reduzir o viés e espalhamento dos dados gerados pelos sensores em verificação.

Para verificar se os coeficientes são eficientes no ajuste de um período amostral diferente daquele em que foram determinados, dados coletados durante períodos estáveis que ocorreram em data pretérita e futura ao PEC PEA 1 e PEA 2 - foram verificados estatisticamente. Houve redução de viés e erro (REQM) para ambos os períodos e para os dois tipos de sensor (Tabela 4). Para o período PEA 1 do sensor EP, o viés passou de $-10,23$ para $-0,84$. Para o PEA 2, de forma similar, passou de $-10,07$ para $-0,76$ (Tabela 4).

Para verificar se o emprego dos coeficientes de ajuste obtidos em um período estável é capaz de ajustar um período instável (em sua maior parte do tempo) o período PIA foi analisado (células em destaque na Tabela 4). Os resultados do viés e REQM mostram redução significativa de seus valores em ambos os sensores, semelhante ao evidenciado para períodos estáveis. De forma complementar, o erro médio absoluto (EMA) e o erro percentual relativo médio (EPMA) foram calculados para todos os períodos. O erro médio absoluto (EMA) para o sensor EP (KZ) reduziu de $7,99(3,71)$ para $0,85(0,54)$. O EPMA para o sensor EP (KZ) reduziu em uma ordem de grandeza, passando de $2,30 \%(1,07 \%)$ para $0,23 \%(0,14 \%)$.
Estes resultados mostram que os coeficientes calculados não são apenas eficientes para ajustar o período de dados em que eles foram determinados mas também são eficientes para ajustar dados em períodos diferentes. Em particular, indica que medições de OLD armazenadas em bancos de dados podem ser corrigidas após o cálculo dos coeficientes de ajuste pelo método aqui proposto.

A Figura 6 apresenta os gráficos de correlação entre o padrão e os sensores EP e KZ. Na Figura 6a fica evidente a presença do viés nos valores obtidos pelo sensor EP utilizando os coeficientes da Rede SONDA, que quando substituídos pelos coeficientes calculados aqui é minimizado (Fig. 6b). O mesmo ocorre com o sensor KZ nas Figuras 6c e $6 \mathrm{~d}$.

Os valores do coeficiente angular das retas de correlação (B) mostraram alterações desprezíveis com a alteração nos coeficientes, passando de 1,022 (0,990) para 0,998 $(0,999)$ no caso do sensor EP $(\mathrm{KZ})$.

\section{Conclusões}

Os coeficientes de correção refletem as características particulares dos pirgeômetros, pois são relacionados ao comportamento dos materiais que constituem o instrumento. Pequenas alterações nas propriedades físicas da termopilha ou do domo de um pigeômetro afetam as medidas de OLD e portanto valores dos coeficientes de correção precisam ser calculados. Cada sensor é único no momento de sua fabricação e detentor de valores de coeficientes próprios. Ainda, os materiais destes sensores apresentam diferentes graus de deterioração ao longo de sua vida útil, relacionados com as diversas condições de intempéries impostas pelo meio ambiente. Portanto, os coeficientes de ajuste necessitam ser recalculados periodicamente como está estabelecido nas normas da Baseline Surface Radiation Network.

Para garantir a confiabilidade dos dados produzidos por pirgeômetros é necessário avaliar constantemente as

Tabela 4 - Viés, raíz quadrada do erro quadrático médio (REQM), erro médio absoluto (EMA) e erro percentual relativo médio (EPMA) calculados para os sensores EP e KZ relativos aos valores produzidos pelo sensor padrão.

\begin{tabular}{|c|c|c|c|c|c|}
\hline & & \multicolumn{2}{|c|}{ PIR 33495F3 (EP) } & \multicolumn{2}{|c|}{ CGR4 $120512(\mathrm{KZ})$} \\
\hline & & $\mathrm{k}_{\mathrm{s}} \mathrm{SONDA}$ & $\mathrm{k}_{\mathrm{s}}$ Calc. & $\mathrm{k}_{\mathrm{s}} \mathrm{Fab}$ & $\mathrm{k}_{\mathrm{s}}$ Calc. \\
\hline \multirow[t]{2}{*}{ PEC } & $\operatorname{VIES}\left(\mathrm{w} / \mathrm{m}^{2}\right)$ & $-8,95$ & $-0,002$ & 4,53 & $-0,003$ \\
\hline & $\operatorname{REQM}\left(\mathrm{w} / \mathrm{m}^{2}\right)$ & 9,02 & 0,43 & 4,71 & 0,31 \\
\hline \multirow[t]{2}{*}{ PEA1 } & $\operatorname{VIES}\left(\mathrm{w} / \mathrm{m}^{2}\right)$ & $-10,23$ & $-0,84$ & 4,30 & 0,02 \\
\hline & $\operatorname{REQM}\left(\mathrm{w} / \mathrm{m}^{2}\right)$ & 10,34 & 0,91 & 4,44 & 0,21 \\
\hline \multirow[t]{2}{*}{ PEA2 } & $\operatorname{VIES}\left(\mathrm{w} / \mathrm{m}^{2}\right)$ & $-10,07$ & $-0,76$ & 4,64 & $-0,06$ \\
\hline & $\operatorname{REQM}\left(\mathrm{w} / \mathrm{m}^{2}\right)$ & 10,22 & 0,92 & 4,94 & 0,52 \\
\hline \multirow[t]{4}{*}{ PIA } & $\operatorname{VIES}\left(\mathrm{w} / \mathrm{m}^{2}\right)$ & $-7,99$ & 0,50 & 3,53 & 0,27 \\
\hline & $\operatorname{REQM}\left(\mathrm{w} / \mathrm{m}^{2}\right)$ & 8,33 & 1,43 & 4,11 & 1,21 \\
\hline & $\operatorname{EMA}\left(\mathrm{w} / \mathrm{m}^{2}\right)$ & 7,99 & 0,85 & 3,71 & 0,54 \\
\hline & EPMA (\%) & 2,30 & 0,23 & 1,07 & 0,14 \\
\hline
\end{tabular}



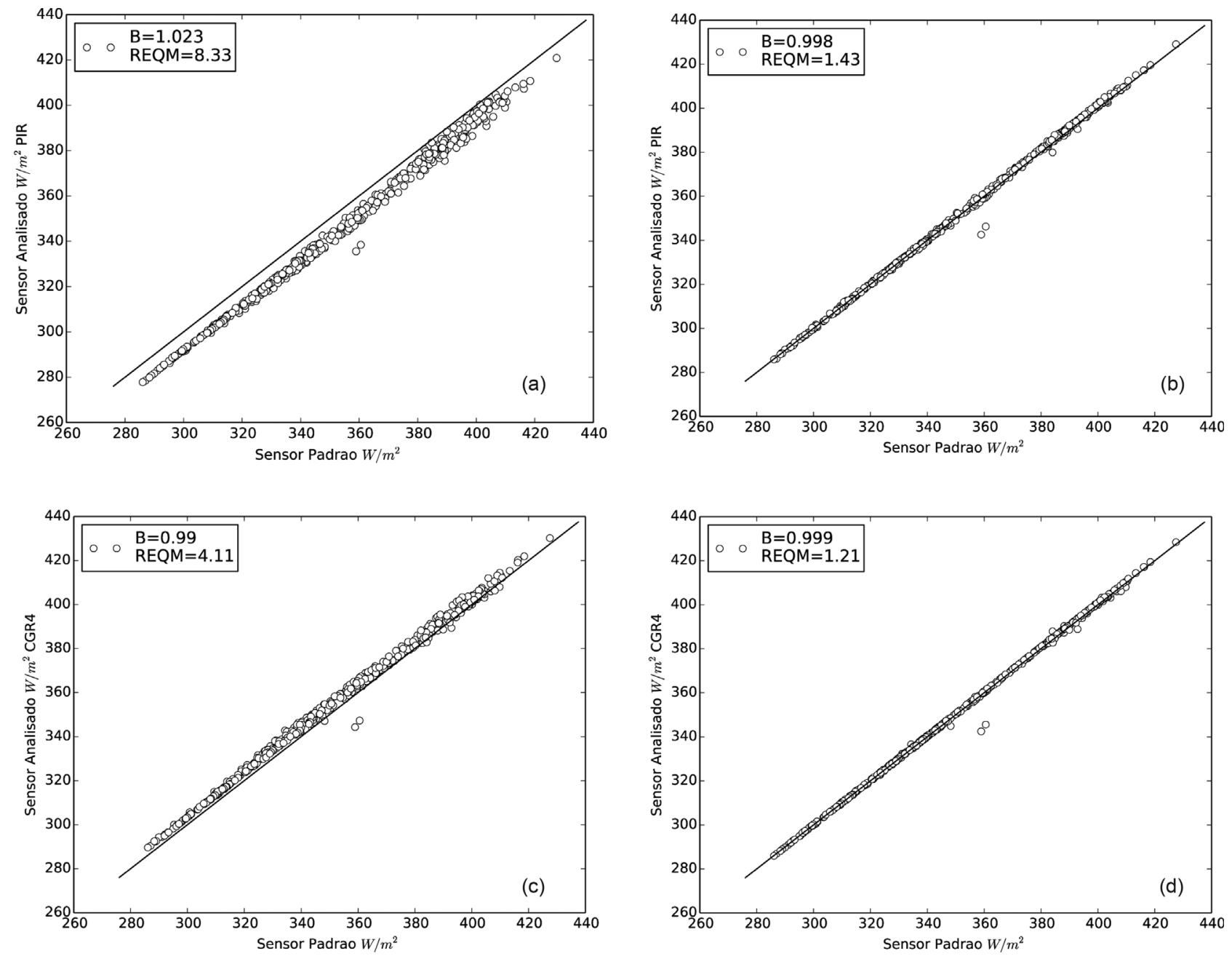

Figura 6 - Diagrama de dispersão da radiação de onda longa descendente medida pelo sensor padrão e pelos sensores em análise utilizando diferentes coeficientes ks (Tabela 3): a) sensor EP (ks da rede SONDA), b) sensor EP (ks calculado neste estudo), c) sensor KZ (ks fabricante), e d) sensor KZ (ks calculados neste estudo). Na legenda canto esquerdo superior, B e REQM são respectivamente, o coeficiente angular da reta e a raiz quadrada do erro quadrático médio.

séries temporais produzidas. Entretanto, não existe uma metodologia definida e consolidada na comunidade científica que esteja disponível para realizar essas análises e possíveis correções necessárias (REDA et al., 2002; Philipona et al., 2001; Gröbner e Wacker, 2012).

Os resultados apresentados mostram que o método utilizado é eficiente para o cálculo dos coeficientes de ajuste e que estes são capazes de ajustar períodos observados em data pretérita ou futura, sejam eles de valores de irradiância com comportamento estável ou instável. Isto indica que será possível corrigir séries históricas de OLD depositadas em bancos de dados. Importante salientar que a correção destes dados históricos somente será possível caso estejam armazenados os valores gerados pelos termistores (temperatura do domo e do corpo do sensor) e pela termopilha, para cada medida. Dessa forma, é recomendado que estes valores não sejam descartados.

\section{Referências}

ALBRECHT, B.; COX, S. Procedures for improving pyrgeometer performance. Journal of Applied Meteorology 16, 188197. 1977.

DIAS NETO, J. Caracterização de pirgeômetros e controle de qualidade dos dados medidos. 2014. 90 p. (sid.inpe.br/mtc-m21b/2014/05.12.18.31-TDI). Dissertação (Mestrado em Meteorologia) - Instituto Nacional de Pesquisas Espaciais (INPE), São José dos Campos, 2014. Disponível em: http://urlib.net/8JMKD3MGP5W34M/3GA8ETS.

DICKEY, T.; MANOV, D.; WELLER, R.; SIEGEL, D. Determination of longwave heat flux at the air-sea interface using measurements from buoy platforms. Journal of Atmospheric and Oceanic Technology 11, 1057-1078. 1994.

DUARTE, H.F.; DIAS, N.L.; MAGGIOTTO, S.R. Assessing daytime downward longwave radiation estimates for clear and cloudy skies in southern Brazil. Agricultural and forest meteorology 139, 171-181. 2006. 
GRÖBNER, J.; WACKER, S. Pyrgeometer Calibration Procedure at the PMOD/WRC-IRS. Davos: [s.n.], 2012. 13 p.

HARRIES, J. E.; BRINDLEY, H. E.; SAGOO, P.J.; BANTGES, R. J. Increases in greenhouse forcing inferred from the outgoing longwave radiation spectra of the earth in 1970 and 1997. Nature 410, 355-357. 2001.

KREYSZIG, E.; Advanced Engineering Mathenatics. Edição 9. Ohio, Columbus, EUA. John Wiley \& Sons, Inc, 2006. 1094p. ISBN 978-0-471-48885-9.

MCARTHUR, L. J. B. World Climate Research ProgrammeBaseline Surface Radiation Network (BSRN)-Operations Manual Version 2.1. Segunda edição. Downsview, Ontario, Canada: Experimental Studies Division, Atmospheric Environment Service, 2005. 188 p.

PEREIRA, E.; LIMA, J. H. G. Solar and wind energy resource assessment in Brazil. Primeira edição. São José dos Campos: [s.n.], 2008. 100 p. ISBN 978-85-17-00038-6.

PHILIPONA, R.; FROHLICH, C.; BETZ, C.; Characterization of pyrgeometers and the accuracy of atmospheric long-wave radiation measurements. Applied Optics 34, 1598-1605. 1995.

PHILIPONA, R.; DUTTON, E. G.; STOFFEL, T.; MICHALSKY, J.; REDA, I.; STIFTER, A.; WENDLING, P.; WOOD, N.; CLOUGH, S. A.; MLAWER, E. J.; ANDERSON, G.; REVERCOMB, H. E.; SHUPPERT, T. R. Atmospheric longwave irradiance uncertainty:
Pyrgeometers compared to an absolute sky-scanning radiometer, atmospheric emitted radiance interferometer, and radiative transfer model calculations. Journal of Geophysical Resarch, v. 106, n. D22, p. 28,129-28,141, 2001.

REDA, I.; HICKEY, J. R.; STOFFEL, T.; MYERS, D. Pyrgeometer calibration at the national renewable energy laboratory (nrel). Journal of Atmospheric and Solar-Terrestrial Physics, v. 64, p. 1623-1629, 2002.

SONDA. Informações sobre o projeto http://sonda.ccst.inpe.br/. Acesso em: 3 de fev. de 14.

THOMAZ, J. C. J. Perspectiva da gestão/administração de sistemas integrados de observação terrestre. 2012. 53p. Monografia - Fundação Getúlio Vargas, São José dos Campos, 2012.

TETT, S.F.; STOTT, P.A.; ALLEN, M.R.; INGRAM, W.J.; MITCHELL, J.F. Causes of twentieth-century temperature change near the earth's surface. Nature 399, 569-572. 1999.

TRENBERTH, K.E.; MOORE, B.; KARL, T.R.; NOBRE, C.; Monitoring and prediction of the earth's climate: A future perspective. Journal of climate 19, 5001-5008. 2006.

WEBSTER, P.J.; LUKAS, R.; Toga coare: The coupled oceanatmosphere response experiment. Bulletin of the American Meteorological Society 73, 1377-1416. 1992.

All the contents of this journal, except where otherwise noted, is licensed under a Creative Commons Attribution License CC-BY. 